\title{
A NEW INEQUALITY FOR COMPLEX-VALUED POLYNOMIAL FUNCTIONS
}

\author{
THEMISTOCLES M. RASSIAS
}

ABstraCt. Let $f_{1}, f_{2}, \ldots, f_{n}: \mathbf{C} \rightarrow \mathbf{C}$ be complex-valued polynomial functions of degrees $d_{1}, d_{2}, \ldots, d_{n}$, respectively, of a complex variable $z$. Then

$$
M_{f_{1}} M_{f_{2}} \cdots M_{f_{n}} \geq M_{f_{1} f_{2} \cdots f_{n}} \geq k M_{f_{1}} M_{f_{2}} \cdots M_{f_{n}}
$$

where

$$
k=\left(\sin \frac{2}{n} \frac{\pi}{8 d_{1}}\right)^{d_{1}}\left(\sin \frac{2}{n} \frac{\pi}{8 d_{2}}\right)^{d_{2}} \ldots\left(\sin \frac{2}{n} \frac{\pi}{8 d_{n}}\right)^{d_{n}} .
$$

Let $\mathbf{C}$ be the field of complex numbers and $f: \mathbf{C} \rightarrow \mathbf{C}$ be a complex-valued polynomial function of a complex variable $z$. Define $M_{f}=\operatorname{Max}_{|z|=1}|f(z)|$.

THEOREM. Let $f_{1}, f_{2}, \ldots, f_{n}: \mathbf{C} \rightarrow \mathbf{C}$ be polynomial functions of degrees $d_{1}, d_{2}$, $\ldots, d_{n}$, respectively, of a complex variable $z$. Then

$$
M_{f_{1}} \cdot M_{f_{2}} \cdots M_{f_{n}} \geq M_{f_{1} \cdot f_{2} \cdots f_{n}} \geq k \cdot M_{f_{1}} \cdot M_{f_{2}} \cdots M_{f_{n}}
$$

where

$$
k=\left(\sin \frac{2}{n} \frac{\pi}{8 d_{1}}\right)^{d_{1}}\left(\sin \frac{2}{n} \frac{\pi}{8 d_{2}}\right)^{d_{2}} \ldots\left(\sin \frac{2}{n} \frac{\pi}{8 d_{n}}\right)^{d_{n}} .
$$

ProOF. Of course the left inequality is obvious.

Let $f: \mathbf{C} \rightarrow \mathbf{C}$ be an arbitrary polynomial function of degree $d$ with leading coefficient one, among the given functions $\left\{f_{i}\right\}$. Then we can factor $f(z)$ in the form

$$
f(z)=\left(z-z_{1}\right)\left(z-z_{2}\right) \cdots\left(z-z_{d}\right)
$$

so that

$$
\begin{aligned}
M_{f} & =\operatorname{Max}_{|z|=1}|f(z)|=\underset{|z|=1}{\operatorname{Max}}\left|z-z_{1}\right|\left|z-z_{2}\right| \cdots\left|z-z_{d}\right| \\
& \leq\left(1+\left|z_{1}\right|\right)\left(1+\left|z_{2}\right|\right) \cdots\left(1+\left|z_{d}\right|\right) .
\end{aligned}
$$

We will prove that for each $\varepsilon>0$ and $n>0$, we have for most $\theta$ that

$$
\left|f\left(e^{i \theta}\right)\right|=\left|e^{i \theta}-z_{1}\right|\left|e^{i \theta}-z_{2}\right| \cdots\left|e^{i \theta}-z_{d}\right| \geq M_{f} \cdot\left(\sin \frac{2}{n} \frac{\pi}{8 d+\varepsilon}\right)^{d} .
$$

However by (2), we see that it is enough to show that

$$
\frac{\left|e^{i \theta}-z_{1}\right|\left|e^{i \theta}-z_{2}\right| \cdots\left|e^{i \theta}-z_{d}\right|}{\left(1+\left|z_{1}\right|\right)\left(1+\left|z_{2}\right|\right) \cdots\left(1+\left|z_{d}\right|\right)} \geq\left(\sin \frac{2}{n} \frac{\pi}{8 d+\varepsilon}\right)^{d}
$$

Received by the editors June 11, 1984 and, in revised form, June 12, 1985.

1980 Mathematics Subject Classification. Primary 30A10; Secondary 30C10.

Key words and phrases. Complex polynomial function, connected subset, length, inequalities. 
for all values of $\theta$ in the complement of the union $U_{d}$ of intervals on $[-\pi, \pi]$ with total length $l\left(U_{d}\right) \leq(2 / n) 8 d \pi /(8 d+\varepsilon)$. Let $z$ be an arbitrary element in $\left\{z_{i}\right\}$ and set $z=r e^{i a}, r>0$, and $\phi=(2 / n) \pi /(8 d+\varepsilon)$. Then for $\theta$ satisfying the inequality $2 \phi \leq|\theta| \leq \pi-2 \phi$, we have $\left(\rho=2 r /\left(r^{2}+1\right)<1\right)$

$$
\begin{aligned}
\frac{\left|1+r e^{i \theta}\right|^{2}}{(1+r)^{2}} & =\frac{1+r^{2}+2 r \cos \theta}{1+r^{2}+2 r} \geq \frac{1+r^{2}-2 r \cos 2 \phi}{1+r^{2}+2 r} \\
& =\frac{1-\left(2 r /\left(1+r^{2}\right)\right) \cos 2 \phi}{1+\left(2 r /\left(1+r^{2}\right)\right)}=\frac{1-\rho \cos 2 \phi}{1+\rho} \geq \frac{1-\cos 2 \phi}{2}=\sin ^{2} \phi .
\end{aligned}
$$

Thus $\left|1+r e^{i \theta}\right| /(1+r) \geq \sin \phi$ for all values of $\theta$ in the complement of the union $V$ of intervals with total length $l(V) \leq 8 \phi$. Therefore, on a similar set we have that

$$
\left|e^{i \theta}-r e^{i a}\right| /\left(1+\left|r e^{i a}\right|\right) \geq \sin \phi
$$

and so (4) follows. Applying the estimate (2) for each polynomial function $f_{i}$ of degree $d_{i}$, we obtain $n$ inequalities of the form (3) (with $f=f_{1}, f_{2}, \ldots, f_{n}$ ), with each holding for all values of $\theta$ in the complement of the union $U_{d_{i}}$ of intervals with total length

$$
l\left(U_{d_{i}}\right) \leq(2 / n) 8 d_{i} \pi /\left(8 d_{i}+\varepsilon\right),
$$

respectively, for $i=1,2, \ldots, n$.

We add this for all $n$, and deduce that all $n$ inequalities (3) hold for some

$$
\beta \in[-\pi, \pi]-\bigcup_{i=1}^{n} U_{d_{i}} .
$$

Therefore

$$
\begin{aligned}
M_{f_{1} \cdot f_{2} \cdots f_{n}} \geq & \left|f_{1}\left(e^{i \beta}\right) \cdot f_{2}\left(e^{i \beta}\right) \cdots f_{n}\left(e^{i \beta}\right)\right| \\
= & \left|f_{1}\left(e^{i \beta}\right)\right|\left|f_{2}\left(e^{i \beta}\right)\right| \cdots\left|f_{n}\left(e^{i \beta}\right)\right| \geq M_{f_{1}} \cdot M_{f_{2}} \cdots M_{f_{n}} \\
& \cdot\left(\sin \frac{2}{n} \frac{\pi}{8 d_{1}+\varepsilon}\right)^{d_{1}}\left(\sin \frac{2}{n} \frac{\pi}{8 d_{2}+\varepsilon}\right)^{d_{2}} \cdots\left(\sin \frac{2}{n} \frac{\pi}{8 d_{n}+\varepsilon}\right)^{d_{n}} .
\end{aligned}
$$

Taking the limit as $\varepsilon \rightarrow 0$ (6) implies

$$
M_{f_{1} f_{2} \cdots f_{n}} \geq k \cdot M_{f_{1}} M_{f_{2}} \cdots M_{f_{n}}
$$

where $k$ satisfies (1).

REMARK. See [1-5] for other results and some research problems concerning inequalities on the derivative of a complex-valued function.

ACKNOWLEDGEMENT. I would like to express my thanks to the referee for helpful suggestions.

\section{REFERENCES}

1. Th. M. Rassias, On the derivative of a complex valued function, Bull. Inst. Math. Acad. Sinica 12 (1984), 423-425.

2. G. M. Rassias, J. M. Rassias, and Th. M. Rassia, A counterexample to a conjecture by $P$. Erdös, Proc. Japan Acad. Sci. Ser. A 53 (1977), 119-121.

3. S. Smale, On the efficiency of algorithms of analysis, Bull. Amer. Math. Soc. (N.S.) 13 (1985), 87-121. 
4. (N.S.) 4 (1981), 1-36.

5. M. J. Todd, Polynomial expected behavior of a privoting algorithm for linear complementary and linear programming problems, Technical Rep. No. 595, School of Operations Research and Industrial Engineering, Cornell Univ., Ithaca, N. Y., 1983.

Current address: 4 Zagoras Street, Paradissos, Amaroussion, 15125 Athens, Greece 